\title{
Entanglement entropy, black holes and holography
}

\author{
Roman V. Buniy * and Stephen D. H. Hsu \\ Institute of Theoretical Science \\ University of Oregon, Eugene, OR 97403
}

\begin{abstract}
We observe that the entanglement entropy resulting from tracing over a subregion of an initially pure state can grow faster than the surface area of the subregion (indeed, proportional to the volume), in contrast to examples studied previously. The pure states with this property have longrange correlations between interior and exterior modes and are constructed by purification of the desired density matrix. We show that imposing a no-gravitational collapse condition on the pure state is sufficient to exclude faster than area law entropy scaling. This observation leads to an interpretation of holography as an upper bound on the realizable entropy (entanglement or von Neumann) of a region, rather than on the dimension of its Hilbert space.
\end{abstract}

Black holes radiate [1] and have entropy [2]. The nature of this entropy is one of the great mysteries of modern physics, especially due to its non-extensive nature: it scales as the area of the black hole (in Planck units), rather than its volume. This peculiar property has led to the holographic conjecture 3, 4] proposing that the number of degrees of freedom in any region of our universe grows only as the area of its boundary. (See [5] for a review and discussion of covariant generalizations of this idea.) The AdS/CFT correspondence [6] is an explicit realization of holography.

The entropy of a thermodynamic system is the logarithm of the number of the available microstates of the system, subject to some macroscopic constraints such as fixed total energy. In certain string theory black holes, these states have been counted explicitly [7, 8]. It has also been proposed that black hole entropy is simply the entropy of quantum entanglement between the causally disconnected interior and exterior of the hole. A description of black hole radiance as originating from entanglement has been known for some time [9]. Starting with a pure state $|\psi\rangle$, and tracing over a subspace (analogous to the black hole volume), one obtains a density matrix with non-zero von Neumann entropy (also known as entanglement entropy). When $|\psi\rangle$ is the ground state of a local quantum field theory (or, more generally, any state with short range quantum correlations), the resulting entropy also exhibits area scaling 10, 11, 12, 13, 14]. Recent work [15] argues that even the string theory microstate entropy can be attributed to entanglement.

In this note we observe that the entanglement entropy does not necessarily scale as the area of the region which is traced over. Indeed, it is easy to obtain pure states which lead to maximal entropy, scaling as the volume. Such states have, as one might expect, long range correlations between interior and exterior modes. Entanglement entropy which scales like volume would seem to contradict the usual area law results for black holes. We show that this contradiction is evaded once gravitational ef-

\footnotetext{
*Electronic address: roman@uoregon.edu

${ }^{\dagger}$ Electronic address: hsu@duende.uoregon.edu
}

fects are taken into account: the maximal entropy states are subject to gravitational collapse. If one requires that their construction not produce a black hole larger than the original fiducial region, area bounds are recovered. This line of reasoning suggests a new, less radical, interpretation of holography which does not require the excision of most of the states in Hilbert space. Instead, holographic bounds can be interpreted as bounds on realizable entanglement entropy in the presence of gravity.

We adopt the following notation in our discussion. The pure state describing the entire system is $\left|\psi_{\mathrm{AB}}\right\rangle$. The subregion over which we trace is $\mathrm{B}$, and has volume $V$ and radius of roughly $R$. We assume an ultraviolet regulator of order the Planck scale and express dimensional quantities in Planck units. We take the Hilbert space $\mathcal{H}_{\mathrm{B}}$ to be that of the QFT modes restricted to region $\mathrm{B}$, so $\operatorname{dim}\left(\mathcal{H}_{\mathrm{B}}\right)=N=c^{V}$ for some constant $c$. (For qubits, or individual spins on a lattice, $c=2$.) The exterior region is denoted by $\mathrm{A}$ and has Hilbert space $\mathcal{H}_{\mathrm{A}}$.

We also remind the reader of the Schmidt decomposition theorem [16]. Suppose $\left|\psi_{\mathrm{AB}}\right\rangle$ is a pure state of a composite system $\mathrm{AB}$. Then there exist orthonormal states $\left|\psi_{\mathrm{A}}^{(n)}\right\rangle$ for system A and $\left|\psi_{\mathrm{B}}^{(n)}\right\rangle$ for system B such that

$$
\left|\psi_{\mathrm{AB}}\right\rangle=\sum_{n} \lambda_{n}^{\frac{1}{2}}\left|\psi_{\mathrm{A}}^{(n)}\right\rangle\left|\psi_{\mathrm{B}}^{(n)}\right\rangle
$$

where $\lambda_{n}^{\frac{1}{2}}$ are nonnegative real numbers satisfying $\sum_{n} \lambda_{n}=1$. This is a simple consequence of the singular value decomposition theorem. Note that the dimensionalities of $\mathcal{H}_{\mathrm{A}}$ and $\mathcal{H}_{\mathrm{B}}$ might be very different, and that the range over which the sum in Eq. (11) runs is determined by the smaller Hilbert space.

\section{Maximum entropy construction}

We first ignore gravitational effects and let $\mathrm{B}$ be an imaginary subvolume (no black holes yet). We work backwards by first choosing the density matrix $\rho_{\mathrm{B}}$ which maximizes entropy:

$$
\rho_{\mathrm{B}}=N^{-1} \sum_{n=1}^{N}\left|\psi_{\mathrm{B}}^{(n)}\right\rangle\left\langle\psi_{\mathrm{B}}^{(n)}\right| .
$$


This results in entanglement entropy

$$
S_{\mathrm{B}}=\ln N=V \ln c,
$$

which is maximal. The complementary density matrix, which describes the mixed state resulting from tracing over the interior region $\mathrm{B}$, is

$$
\rho_{\mathrm{A}}=N^{-1} \sum_{n=1}^{N}\left|\psi_{\mathrm{A}}^{(n)}\right\rangle\left\langle\psi_{\mathrm{A}}^{(n)}\right| .
$$

$\rho_{\mathrm{A}}$ has the same (non-zero) eigenvalues as $\rho_{\mathrm{B}}$, and hence the same entropy, $S_{A}=S_{B}$. The $\left|\psi_{\mathrm{A}}^{(n)}\right\rangle$ and $\left|\psi_{\mathrm{B}}^{(n)}\right\rangle$ are orthogonal vectors forming the Schmidt basis.

We can construct a pure state

$$
\left|\psi_{\mathrm{AB}}\right\rangle=N^{-\frac{1}{2}} \sum_{n=1}^{N}\left|\psi_{\mathrm{A}}^{(n)}\right\rangle\left|\psi_{\mathrm{B}}^{(n)}\right\rangle
$$

which, upon tracing over $\mathrm{B}$, yields the desired $\rho_{\mathrm{A}}$, and hence volume behavior of the entropy. (This is possible for any desired $\rho$, which can be seen from the Schmidt decomposition. The procedure is known as "purification" of a mixed state.) There are many such states, as an $i$-dependent phase factor in the sum does not affect the resulting density matrix. All such states exhibit substantial long range correlations between interior and exterior modes. This is easy to see, since there are only $c^{A}$ states localized near the boundary; correlations between $N \sim c^{V}$ interior and exterior states must have typical range at least as large as $R$. In fact, we expect any states $\left|\psi_{\mathrm{AB}}\right\rangle$ with sufficiently large quantum correlation lengths to yield entropies which scale like volume [13, 14].

In the purification procedure, the Hilbert space $\mathcal{H}_{\mathrm{A}}$ is taken isomorphic to $\mathcal{H}_{\mathrm{B}}$, but otherwise the physical nature of the A states is unspecified. They could be QFT degrees of freedom in the exterior or abstract qubits (spins) whose orientation states form $\mathcal{H}_{\mathrm{A}}$. We assume the latter case below, which means that the A components of $\left|\psi_{\mathrm{AB}}\right\rangle$ can be manipulated (moved about) without energetic consequences.

\section{Gedanken construction of black hole in region $B$}

Consider the consequences of an entanglement entropy $S$ which grows like $V$. It seems reasonable to assume that the entanglement entropy $S$ contributes to the Bekenstein-Hawking entropy $S_{\mathrm{BH}}$. In [15] it is argued that $S_{\mathrm{BH}}=S$, although one might also imagine that $S_{\mathrm{BH}}=S+S^{\prime}$, where $S^{\prime}$ is an additional (positive) source of entropy. Additional sources of entropy $S^{\prime}$ might arise from coarse graining - for example, characterizing the location of the horizon in a classical way despite small Planck-length fluctuations in its position. However, these are likely to exhibit area scaling and do not affect our arguments below.

Let the B region collapse into a black hole of volume $V$. Let the A states be abstract qubits, which we spread thinly over the rest of the universe (their correlations with the B states are independent of their position, so are unaffected by the spreading). A semi-classical calculation is then sufficient to determine the entropy of the black hole. This yields the usual Bekenstein-Hawking entropy $S_{\mathrm{BH}}$, which scales like the area, in contradiction with an entanglement entropy $S$ which scales as $V$.

\section{Holographic resolution}

The contradiction is resolved by noting that gravitational collapse limits the number of states $N$ we can use in our construction of $\left|\psi_{\mathrm{AB}}\right\rangle$. Note that this is subtly different from limiting the actual size of $\mathcal{H}_{\mathrm{B}}$.

In the gedanken construction, it is reasonable to require that $\left|\psi_{\mathrm{AB}}\right\rangle$ not have already undergone collapse to a black hole larger than the region $\mathrm{B}$.

Let the Hamiltonian be $H=H_{\mathrm{A}}+H_{\mathrm{B}}+H_{\mathrm{AB}}$. We take the A states to be abstract qubits, and set $H_{\mathrm{A}}=0$ by not allowing any interactions between them. Since the desired state $\left|\psi_{\mathrm{AB}}\right\rangle$ is a pure state, we can build it using unitary transformations on any initial pure state. It is well-known in quantum information theory that a general unitary transformation can be efficiently implemented as the product of primitive manipulations, each involving only a small number of qubits [16]. Therefore, in each step of the construction of $\left|\psi_{\mathrm{AB}}\right\rangle$ the interaction term $H_{\mathrm{AB}}$ need not be large and can neglected relative to $H_{\mathrm{B}}$ for our purposes.

Then

$$
\left\langle\psi_{\mathrm{AB}}|H| \psi_{\mathrm{AB}}\right\rangle=N^{-1} \sum_{n=1}^{N}\left\langle\psi_{\mathrm{B}}^{(n)}\left|H_{\mathrm{B}}\right| \psi_{\mathrm{B}}^{(n)}\right\rangle
$$

which is the average of expectation values $\left\langle H_{\mathrm{B}}\right\rangle$ in states included in the $\left|\psi_{\mathrm{AB}}\right\rangle$ superposition.

The no-gravitational collapse requirement then implies (roughly) that 17]

$$
\left\langle\psi_{\mathrm{AB}}\left|H_{\mathrm{B}}\right| \psi_{\mathrm{AB}}\right\rangle<R
$$

Now, if one excludes states from the Hilbert space whose energies are so large that they would have already caused gravitational collapse, one obtains $\ln N<A^{\frac{3}{4}}$, as originally deduced by 't Hooft [3]. 't Hooft replaces the system under study with a thermal one. The number of states of a system with constant total energy $E$ is given to high accuracy by the thermal result in the large volume limit (recall the microcanonical ensemble in statistical mechanics). Given a thermal region of radius $R$ and temperature $T$, we have $S_{\mathrm{th}} \sim T^{3} R^{3}$ and $E \sim T^{4} R^{3}$. Requiring $E<R$ then implies $S_{\mathrm{th}}<R^{\frac{3}{2}} \sim A^{\frac{3}{4}}$. These relations also imply

$$
E R \sim S_{\mathrm{th}}^{\frac{4}{3}} \sim(\ln \nu)^{\frac{4}{3}},
$$

where $\nu$ is the multiplicity of states with total energy $E$. We stress that the thermal replacement is just a calculational trick: temperature plays no role in our results, and Eq. (8) can be obtained also by direct counting. 
Avoiding collapse to a black hole larger than $R$ requires that we cut off the sum in (5) at of order $N \sim \exp A^{\frac{3}{4}}$, well before the maximum $N=c^{V}$, which prevents our construction of states $\left|\psi_{\mathrm{AB}}\right\rangle$ whose density matrices violate the area bound.

This conclusion is more general than our original construction. Previously we began with states of maximal entropy and only subsequently imposed the nogravitational collapse condition. In the following we maximize the entropy subject to the collapse condition (the two steps do not commute). The resulting density matrices are canonical ensembles, with Boltzmann probabilities, in contrast to the equal probabilities in (2) and (4). Nevertheless, the resulting upper bound on entropy scales only as $A^{\frac{3}{4}}$.

Let $\left|\psi_{\mathrm{AB}}\right\rangle$ be an arbitrary pure state, and consider the question of gravitational collapse in the region $\mathrm{B}$. Whether collapse occurs depends on local properties in $\mathrm{B}$, so we can trace over the A degrees of freedom and consider the resulting density matrix

$$
\rho_{\mathrm{B}}=\sum_{n=1}^{N} \lambda_{n}\left|\psi_{\mathrm{B}}^{(n)}\right\rangle\left\langle\psi_{\mathrm{B}}^{(n)}\right| .
$$

At this point the bound we derive on $S$ could either be interpreted as a bound on entanglement entropy, or simply a bound on the usual von Neumann entropy of the state which collapses to form the black hole. A reasonable no-collapse criteria is then

$$
\operatorname{tr}\left(\rho_{\mathrm{B}} H_{\mathrm{B}}\right)=\left\langle H_{\mathrm{B}}\right\rangle<R .
$$

Adopting an energy eigenstate basis, we have the condition

$$
\sum_{n=1}^{N} \lambda_{n} E_{\mathrm{B}}^{(n)}<R
$$

where the $\lambda_{n}$ (eigenvalues of $\rho_{\mathrm{B}}$ ) are the probabilities for finding the system in energy eigenstate $n$. Note the requirement that the gravitational field produced by the matter in region B is semi-classical (so that the hoop conjecture or some similar collapse criteria [17] can be applied) may constrain the distribution of $\lambda_{n}$ even more, requiring it to be highly peaked around some central value. We do not impose this condition in our analysis, though it likely strengthens our results.

Let us maximize

$$
S=-\sum_{n} \lambda_{n} \ln \lambda_{n},
$$

where the $\left\{\lambda_{n}\right\}$ are subject to constraints $\sum_{n} \lambda_{n}=1$ and

$$
\sum_{n} \lambda_{n} \epsilon_{n}=A
$$

where $\epsilon_{n}=E_{\mathrm{B}}^{(n)} R$. We impose equality in (13) knowing that, since the density of states grows with energy, the entropy will be maximized when the total energy of the system is also maximal. Using the method of Lagrange multipliers with

$$
\begin{aligned}
\tilde{S}=-\sum_{n} \lambda_{n} \ln \lambda_{n} & +\alpha\left(\sum_{n} \lambda_{n}-1\right) \\
& -\beta\left(\sum_{n} \lambda_{n} \epsilon_{n}-A\right),
\end{aligned}
$$

variations with respect to $\lambda_{n}$ and $\alpha$ give

$$
\lambda_{n}=Z^{-1} e^{-\beta \epsilon_{n}},
$$

where

$$
Z(\beta)=\sum_{n} e^{-\beta \epsilon_{n}}
$$

The resulting entropy,

$$
\tilde{S}(\beta)=\ln Z(\beta)-\beta A,
$$

reaches its maximum at the point $\beta=\beta_{*}$, which is the solution to the equation

$$
Z^{\prime}(\beta)+A Z(\beta)=0 .
$$

It is easy to see that this equation always has one solution. Finally, using Eqs. (12), (15) and (18), we find

$$
\max S=\ln Z\left(\beta_{*}\right)+\beta_{*} A .
$$

We now express the maximum entropy in terms of the density of states. We start by replacing the sum in $Z(\beta)$ by the integral,

$$
Z(\beta)=\int d \epsilon \nu(\epsilon) e^{-\beta \epsilon},
$$

where $\nu(\epsilon)=d n(\epsilon) / d \epsilon$, and evaluate it by the saddle point method. The result is

$$
\ln Z(\beta) \approx \ln \nu\left(\epsilon_{*}\right)-\beta \epsilon_{*},
$$

where $\epsilon=\epsilon_{*}(\beta)$ is the solution of $d \ln \nu(\epsilon) / d \epsilon=\beta$. Eq. (18) becomes $\epsilon_{*}(\beta) \approx A$ with a solution $\beta=\beta_{*}(A)$. From Eq. (19), the maximum entropy is simply

$$
\left.\max S \approx \ln \nu(\epsilon)\right|_{\epsilon=A} .
$$

From Eq. (8), we immediately find

$$
\max S \approx A^{\frac{3}{4}}
$$

Since the maximally entropic system is thermal (see (16)), and the temperature $T=\beta^{-1}$ is determined by the condition (13) that the average total energy be $R$, our result inevitably agrees with 't Hooft's calculation described earlier. One easily generalizes to $d$ dimensions to obtain $A^{(d-1) / d}$ scaling.

Note, we have assumed simple boundary conditions (appropriate to a finite box) in our calculation. While 
this is sufficient to count states which might contribute extensively (i.e., as $V$ ) to the entanglement entropy via long range correlations, it does not properly treat contributions resulting from short range correlations at the boundary of $B$, which are order $A$ 10, 11, 12, 13, 14, 15]. It is interesting that once the gravitational collapse condition is imposed, the maximal contribution of 'bulk' states (those not localized near the boundary) is reduced from volume scaling to $A^{\frac{3}{4}}$, which is smaller than the original $A$ scaling from boundary correlations.

\section{Conclusions}

The holographic conjecture makes the rather strong assertion that states with $\langle i|H| i\rangle$ greater than $R$ simply do not exist in the Hilbert space. This conjecture, however, leads to a number of puzzles. It is unclear what becomes of locality or how unitarity is preserved. Do the high energy states participate in virtual processes? How is the path integral measure modified? Of course the biggest puzzle related to holography is why the universe appears to have $d$ spacetime dimensions if the Hilbert space is only that of a $d-1$ dimensional system.

We suggest an alternative interpretation of black hole entropy bounds. The gravitational collapse condition on $\left|\psi_{\mathrm{AB}}\right\rangle$ places an upper bound on the entanglement (or von Neumann) entropy that can be realized from a region B without forming a black hole larger than B itself. Highly energetic states remain in the theory (they appear explicitly in the maximal entropy states; see Eq. (9) and (15)), but cannot increase the entanglement entropy beyond the area of B in Planck units. Entropy bounds reflect the limitations that gravity imposes on the construction of pure states or density matrices, but do not require a truncation of the Hilbert space itself.

Acknowledgements. - The authors thank A. Zee for useful comments. The authors are supported by the Department of Energy under DE-FG06-85ER40224.
[1] S. W. Hawking, Commun. Math. Phys. 43, 199 (1975).

[2] J. D. Bekenstein, Phys. Rev. D7, 2333 (1973).

[3] G. 't Hooft, gr-qc/9310026

[4] L. Susskind, J. Math. Phys. 36, 6377 (1995), hep-th/9409089.

[5] R. Bousso, Rev. Mod. Phys. 74, 825 (2002), hep-th/0203101.

[6] J. M. Maldacena, Adv. Theor. Math. Phys. 2, 231 (1998), hep-th/9711200.

[7] A. Strominger and C. Vafa, Phys. Lett. B379, 99 (1996), hep-th/9601029.

[8] J. M. Maldacena, A. Strominger and E. Witten, JHEP 12, 002 (1997), hep-th/9711053.

[9] W. Israel, Phys. Lett. A57, 107 (1976).

[10] L. Bombelli, R. Koul, J. Lee and R. Sorkin, Phys. Rev. D 34, 373 (1986).

[11] M. Srednicki, Phys. Rev. Lett. 71, 666 (1993), hep-th/9303048.

[12] L. Susskind and J. Uglum, Phys. Rev. D 50, 2700 (1994) arXiv:hep-th/9401070; C. G. Callan and F. Wilczek, Phys. Lett. B 333, 55 (1994) arXiv:hep-th/9401072; D. Kabat and M. J. Strassler, Phys. Lett. B329, 46 (1994), hep-th/9401125; C. Holzhey, F. Larsen and F. Wilczek, Nucl. Phys. B424, 443 (1994), hep-th/9403108.

[13] R. Brustein, D. H. Oaknin and A. Yarom, Phys. Rev. D 70 (2004) 044043 arXiv:hep-th/0310091; A. Yarom and R. Brustein, Nucl. Phys. B 709, 391 (2005) arXiv:hep-th/0401081.

[14] M. B. Plenio, J. Eisert, J. Dreissig and M. Cramer, Phys. Rev. Lett. 94, 060503 (2005) arXiv:quant-ph/0405142; M. Cramer, J. Eisert, M. B. Plenio and J. Dreissig, arXiv:quant-ph/0505092

[15] R. Brustein, M. Einhorn and A. Yarom, arXiv:hep-th/0508217.

[16] M. A. Nielsen and I. L. Chuang, Quantum Computation and Quantum Information, Cambridge University Press, 2000.

[17] K. S. Thorne, Nonspherical gravitational collapse: A short review, in J. R . Klauder, Magic Without Magic, San Francisco 1972, 231-258; D. M. Eardley and S. B. Giddings, Phys. Rev. D 66, 044011 (2002) arXiv:gr-qc/0201034; S. D. H. Hsu, Phys. Lett. B 555, 92 (2003) arXiv:hep-ph/0203154. 